ANUARIO DE Estudios MEdIEVALES 44/1, enero-junio de 2014, pp. 215-238

ISSN 0066-5061

doi:10.3989/aem.2014.44.1.07

\title{
LA FORMAZIONE DI UN'ARISTOCRAZIA: MONACHE E MONASTERI FEMMINILI A VENEZIA TRA IX E XIII SECOLO ${ }^{1}$
}

\author{
SHAPING AN ARISTOCRACY: NUNS AND NUNNERIES IN VENICE \\ (IX-XIII CENTURIES)
}

\author{
ANNA RAPETTI \\ Università Ca’ Foscari (Venezia)
}

\begin{abstract}
Riassunto: L'articolo si propone di esaminare il processo di definizione dell'identità del ceto dirigente di Venezia nel periodo IX-XIII secolo e il ruolo occupato dalle relazioni tra le famiglie dell'aristocrazia che costituivano quel ceto $\mathrm{e}$ alcuni monasteri femminili. In questa prospettiva, innovativa nell'analisi della formazione dell' aristocrazia di Venezia, si prenderà in esame l'importante monastero femminile di San Zaccaria e si farà inoltre riferimento ad alcuni altri, sorti sulle isole della laguna tra XII e XIII secolo, espressione di un clima religioso e sociale nuovo e particolare. Attraverso l'analisi dei comportamenti adottati dalle famiglie aristocratiche nei confronti di questi monasteri, si dimostra che il ceto dirigente veneziano si servì delle relazioni costanti con le monache come di uno strumento della lotta politica particolarmente utilizzato dalle famiglie dell' aristocrazia della città. Si osserva anche che alcuni monasteri femminili furono a loro volta soggetti attivi nella definizione di un'identità cittadina che coincideva largamente con il suo gruppo dirigente e con l'esercizio del potere.
\end{abstract}

Parole chiave: monachesimo; donne; aristocrazia; Venezia; potere.

\begin{abstract}
This paper examines the processes that shaped the identity of the Venetian aristocracy from the 9th to the 13th centuries and the role played by the relationship between patrician families and some female monasteries. The important female monastery of San Zaccaria and some other nunneries created in the lagoon between the 12th and the 13th centuries are taken into account in the analysis of the formation of Venetian aristocracy from this innovative perspective. By analysing the behaviour of the aristocratic families towards these nunneries, the paper points out how Venetian ruling groups made use of the long-term relationship with nuns as a particularly useful instrument of political competition. The article also shows how some female monasteries were relevant social actors in the process of definition of Venetian identity, largely coinciding with their own ruling groups, and in particular with the aristocracy, and with the exercise of power.
\end{abstract}

Keywords: monasticism; women; aristocracy; Venice; power.

1 Abbreviazioni utilizzate: ASVE $=$ Archivio di Stato di Venezia; DD $=$ Die Urkunden der deutschen Könige und Kaiser, Diplomata; MGH = Monumenta Germaniae Historica. 


\section{SOMMARIO}

1. La difficile formazione di un ceto dirigente.- 2. Monasteri femminili e dogi.- 3. Le politiche di reclutamento delle monache.- 4 . Le nuove fondazioni.- 5. Conclusioni.6. Bibliografia citata.

\section{LA DIFFICILE FORMAZIONE DI UN CETO DIRIGENTE}

Questo articolo vuole proporre una lettura del processo di definizione del ceto dirigente di Venezia nel periodo antecedente la celebre 'serrata' del Maggior Consiglio del 1297, alla luce delle relazioni intercorrenti tra le famiglie dell'aristocrazia vecchia e nuova che facevano parte di quel ceto $-\mathrm{O}$ aspiravano a farne parte- e alcuni monasteri femminili di antica e più recente fondazione. Per i primi si farà riferimento al monastero di San Zaccaria, per i secondi a un gruppo di comunità sorte nelle isole della laguna settentrionale tra la fine del XII e l'inizio del XIII secolo. Partendo dalla considerazione che, nel periodo preso in esame, anche quando il suo orizzonte politico si allargò a una dimensione internazionale, l'aristocrazia veneziana manifestò un interesse costante per le fondazioni femminili della città, a favore delle quali profuse risorse materiali e sociali, si vuole cercare di capire quale fosse la posizione di queste fondazioni in seno a un gruppo dominante mutevole nella composizione e nel profilo, per quale motivo le famiglie conservassero, per un arco di tempo molto lungo, un interesse così vivo per le monache della città, e infine se queste comunità abbiano avuto o meno un ruolo attivo nel processo di consolidamento della nobiltà veneziana. Si vuole cioè valutare quale funzione sociale e forse politica esse abbiano assolto nel corso di quei cinque secoli ${ }^{2}$, durante i quali l'identità statuale veneziana andò formandosi; quell'identità che si sarebbe poi trasformata in un vero e proprio 'mito', accuratamente coltivato dal potere pubblico.

Questa prospettiva di indagine può offrire risultati innovativi e interessanti. Sinora, gli studi sui rapporti tra ceto dirigente veneziano ed enti religiosi si sono concentrati quasi esclusivamente sull'età moderna, cioè su un'epoca in cui esso non solo aveva ormai raggiunto da tempo un'identità di classe, diventando un vero e proprio patriziato, ma in cui vi era anche una netta definizione istituzionale e giuridica di tale identità. Nel periodo qui considerato, invece, la necessità di stabilire chi appartenesse o meno al gruppo di

\footnotetext{
${ }^{2}$ Come scrive K. Lowe, Power and institutional identity, p. 130: "Convents (...) in Venice were not powerless (...) rather, they were deeply and fatally involved in the great game of manipulating and being manipulated by power". La studiosa si riferisce alla Venezia del Rinascimento, ma la stessa considerazione può essere fatta per l'epoca che qui si prende in esame.
} 
vertice, chi avesse diritto o meno di partecipare al governo del ducato, provocò lotte feroci tra famiglie in ascesa e famiglie in declino, in cui furono utilizzati strumenti diversi. In questa lotta la funzione svolta dai monasteri femminili dovette essere di grande rilievo, come dimostra l'interesse costante verso di loro da parte delle diverse famiglie. Essi furono uno dei luoghi in cui la competizione tra i gruppi famigliari poteva stemperarsi, in vista di un'azione di governo meno concorrenziale e più efficace per l'intera città. I cenobi femminili veneziani offrirono l'occasione a un intero ceto, non solo a singole famiglie, di definire la propria appartenenza al ceto dirigente prima che esso si chiudesse in un patriziato ereditario.

Il periodo preso in esame va dall'inizio del IX secolo, quando a Venezia vennero fondati i primi cenobi femminili, alla fine del XIII, quando vi fu un netto, anche se non definitivo, irrigidimento dell'aristocrazia come ceto ereditario, grazie ai provvedimenti istituzionali del 1297. La documentazione di cui si farà uso, una tipologia molto tradizionale rappresentata in maggioranza da documenti notarili e catastici conservati negli archivi dei monasteri ${ }^{3}$, si presenta in larga misura come 'inedita', non essendo stata la storia monastica veneziana dei secoli centrali del medioevo oggetto di studi sistematici. Essa offre dunque ancora oggi ampie possibilità di conseguire risultati originali a proposito del tema qui affrontato. La sua disuguale disponibilità per il lungo arco cronologico considerato -abbondante e abbastanza omogeneamente distribuita nel tempo per San Zaccaria, molto meno numerosa e concentrata a partire dal Duecento per i monasteri della laguna nord-produce inevitabilmente una deformazione, a vantaggio dell'antico monastero benedettino, del quadro dei rapporti tra gli enti e tra questi e i laici, e un'ulteriore difficoltà nella corretta definizione dei diversi ruoli occupati. Se ne deve tenere conto nel valutare la posizione delle monache e delle loro comunità in seno alla società veneziana e nel corso delle trasformazioni istituzionali e politiche che la attraversarono. Resta però il fatto che la maggiore o minore attività di produzione e conservazione delle fonti è un ottimo indicatore della rilevanza sociale ed economica di queste istituzioni e della coscienza della loro centralità; sotto questo profilo, l'abbondante documentazione di alcune -in confronto alla più modesta produzione delle altre- è anch'essa uno degli strumenti di affermazione del loro ruolo e uno dei segnali che ne denunciano la diversa importanza.

I monasteri di donne ebbero fin dai tempi più remoti una posizione di rilievo nel ducato veneziano. Due dei più antichi, tra quelli sorti nel IX secolo nell'area cittadina, erano appunto comunità femminili; si tratta dei cenobi

${ }^{3}$ La documentazione si trova in ASVE, Corporazioni religiose soppresse, ad vocem. Si veda A. Da Mosto, L'Archivio di Stato di Venezia, pp. 1103-1111. 
di San Zaccaria, già menzionato, e di San Lorenzo, entrambi di fondazione privata, le cui prime notizie risalgono rispettivamente all' 829 e all' $853^{4}$. Fin dall'origine essi si conquistarono, per una serie di circostanze di cui si dirà, un prestigio e un rilievo politico e religioso di cui continuarono a godere ininterrottamente, senza diminuzioni sostanziali, anche quando molte altre comunità femminili erano ormai sorte in città e nelle isole della laguna. Di più; la loro preminenza si realizzò in una situazione che divenne ben presto di forte competizione tra fondazioni vecchie e nuove, competizione che nasceva dalla necessità di queste ultime di assicurarsi protezione nelle dispute, sostegno alle proprie politiche e un adeguato incremento patrimoniale. Fu la vivacissima dinamica sociale e demografica del fluido gruppo dirigente cittadino, impegnato nella propria definizione, a offrire alle monache ampie possibilità di trovarsi uno o più benefattori capaci di garantire adeguato sostegno. Il lungo primato soprattutto di San Zaccaria -che pare non venir meno fino al Rinascimentodovrà essere valutato anche in riferimento ad alcuni nuovi monasteri sorti verso la fine del XII secolo prevalentemente nella laguna a nord di Venezia, spesso collegati o almeno influenzati dagli ordini monastici riformati e nuovi, e al rapporto tra questi e le famiglie dell'aristocrazie. Vedremo che molte famiglie in ascesa, che non appartenevano al vecchio gruppo dirigente dominante sino all'avvento del comune, ma aspiravano a entrarvi, consolidarono i legami con queste comunità dando e ricevendo sostegno politico e spesso anche economico.

I monasteri femminili, soprattutto quelli più prestigiosi, come la storiografia ha ampiamente dimostrato, erano al centro di complesse reti di relazioni istituzionali, politiche ed economiche che collegavano le monache alle famiglie di provenienza, alle autorità cittadine, ai benefattori, poi anche alle autorità ecclesiastiche locali e centrali: vescovi, congregazione, papato. Era una trama di rapporti che negava qualsiasi ipotesi di un loro isolamento sociale e materiale, persino nel caso delle fondazioni sorte nelle remote isole della laguna veneziana. Al contrario: i caratteri intrinseci dei cenobi femminili, anzitutto i limiti posti dalle regole o dai rami maschili degli ordini di appartenenza per esempio alla libertà di movimento delle badesse e alla facoltà di amministrare autonomamente i propri beni, li rendevano particolarmente permeabili agli interventi dall'esterno di uomini e famiglie che, offrendo un servizio che colmava i vuoti aperti dalle molte restrizioni poste all'autorità delle badesse, trovavano modo di incrementare il loro prestigio nell'ambito cittadino.

\footnotetext{
${ }^{4}$ Agli stessi anni (819) risalgono le prime notizie di quello maschile, anch'esso di fondazione privata, di S. Ilario: M. Pozza, Per una storia dei monasteri veneziani, pp. 26-29.
} 
Il periodo che si intende considerare si apre con le prime prove politiche, nel IX secolo, di un'élite cittadina di difficile definizione ma nel suo insieme estremamente competitiva, all'interno della quale la concorrenza per il potere era spesso esasperata dal dinamismo economico delle nuove famiglie in ascesa, e dalle difficoltà di altre, di più antiche fortune, a conservare la propria posizione. L'estremo cronologico più basso coincide con la fase in cui tale competizione raggiunse un primo punto di equilibrio grazie al provvedimento legislativo del 1297 noto come 'serrata' del Maggior Consiglio. Il lungo arco di tempo racchiuso entro questi due termini fu caratterizzato dal progressivo, faticosissimo definirsi del ceto dirigente veneziano come vera e propria aristocrazia dotata di un'identità sempre più netta e distinta dagli altri gruppi sociali del ducato. Si trattò di un processo non solo lungo, ma anche particolarmente difficile e contrastato che trovò il proprio esito nella 'serrata', l'evento considerato come spartiacque tra un'aristocrazia dal profilo e dai confini incerti e mutevoli e una nobiltà ereditaria precostituita, un patriziato dominante composto da un preciso numero di famiglie, il quale si sarebbe nettamente separato dagli altri ceti che, pur continuando la competizione per il potere, da quel momento ne sarebbero stati definitivamente esclusi ${ }^{5}$. Ma il Duecento fu ancora un'età di profonda e complessa competizione fra le famiglie più antiche e quelle in ascesa grazie alle loro fortune mercantili. Si vedrà come, fino a quell'epoca, i rapporti con monasteri antichi e recenti potessero essere uno strumento spesso efficace nel processo di costruzione dell'identità delle singole famiglie e di consolidamento della loro appartenenza al sempre fluido gruppo dominante. A loro volta, grazie al patronato delle famiglie eminenti, i monasteri vedevano crescere grandemente prestigio, ricchezza economica e rilevanza sociale e potevano rivendicare privilegi e tradizioni particolari che ne definivano, in ultima analisi, il ruolo sociale all'interno del dogado ${ }^{6}$.

\section{MONASTERI FEMMINILI E DOGI}

Lo stretto legame tra il doge e la comunità di San Zaccaria risaliva alle origini stesse del cenobio, fondato nel IX secolo dal doge Giustiniano Parteciaco o Partecipazio ${ }^{7}$, il quale lo volle constructum prope palacium, cioè

5 Sull'importanza della 'serrata' del 1297 nella formazione di un vero e proprio patriziato ereditario la storiografia è tutt'altro che unanime. Tra i più autorevoli studiosi che hanno messo seriamente in dubbio la fondatezza di questa peraltro più che consolidata tradizione interpretativa vi è S. Chojnacki, La formazione della nobiltà, p. 641.

${ }^{6}$ K. Lowe, Power and Institutional Identity, p. 129, in riferimento al XIV e XV secolo.

${ }^{7}$ M. Pozza, Per una storia dei monasteri veneziani, p. 28. 
edificato accanto al palazzo ducale, come i documenti ripetutamente ricordano ${ }^{8}$. Non solo: a ulteriore dimostrazione che si trattava di un vero e proprio Eigenkloster del doge, Giustiniano stabilì nel suo testamento dell' 829 che la cappella da lui voluta, che avrebbe dovuto accogliere le reliquie di san Marco, sorgesse su un terreno appartenente a San Zaccaria ${ }^{9}$. Uno degli elementi che distinse questa fondazione veneziana fu però il fatto che la comunità rimase legata non alla famiglia del fondatore, il quale del resto si era già privato nell' 829 dei principali diritti sull'ente legati alla fondazione, ma alla massima carica cittadina, che come è noto era elettiva e non ereditaria. La forza di tale legame portò otto dogi, tra il IX e il XII secolo, a farsi seppellire entro le mura monastiche ${ }^{10} \mathrm{e}$ anche alcuni parenti dei dogi vollero esservi sepolti, come Giovanni, figlio di Pietro II Orseolo, e sua moglie, la principessa greca Maria, imparentata con la famiglia imperiale ${ }^{11}$. In questo modo San Zaccaria fu, fino al XII secolo, il luogo deputato alla conservazione delle più sacre memorie cittadine, incarnate dal corpo dei suoi dogi sepolti entro il chiostro; fu non solo il sacrario dei singoli dogi, ma il luogo in cui il cuore politico dello stato veniva preservato e celebrato. Oltre a questo, San Zaccaria fu coinvolto attivamente nell'attività politica ducale, come dimostrano le visite al cenobio compiute dagli imperatori Ottone III nel $1001^{12}$, Enrico III nel $1040^{13}$, Enrico IV nel $1095^{14}$, e forse anche Enrico V nel $1116^{15}$, durante le loro discese in Italia. È dunque evidente che il monastero occupava un ruolo simbolico, che doveva rendersi manifesto anche agli stranieri, e soprattutto a stranieri tanto illustri, di 'rappresentazione visiva' della comunità cittadina; un ruolo fondamentale

8 La specificazione si trova per esempio nei documenti del X secolo di donazione di appezzamenti a Monselice da parte di Ingelfredo, conte Verona, e di Idelburga moglie del conte Adalbergo; originale del primo si trova in ASVE, Corporazioni religiose soppresse, San Zaccaria, b. 20, pergamene, 914 dicembre 2; edizione di entrambi in F. Corner, Ecclesiae Venetae, XIII, pp. 347-348, 352.

9 M. Agazzi, Platea Sancti Marci, p. 13; M. Pozza, Per una storia dei monasteri veneziani, p. 28 .

${ }^{10}$ I. Fees, Le monache di San Zaccaria, p. 7.

${ }^{11}$ G. Diacono, Istoria Veneticorum, pp. 206-11. Di questa domna ductrix bizantina, o meglio dei suoi costumi raffinati e del suo amore per il lusso eccessivo scrive con accenti di riprovazione e condanna Pier Damiani, ricordandone l'abitudine di fare il bagno nell'acqua piovana (e si può facilmente comprendere il motivo di questa predilezione: l'acqua della laguna doveva essere sconsigliata per qualsiasi tipo di abluzione), di riempire la sua stanza di profumi e di portare il cibo alla bocca non con le mani, ma con uno strumento del tutto inusuale, cioè una piccola forchetta d'oro. Naturalmente, stando al racconto del Damiani, Maria fu duramente punita da Dio, che le procurò una morte vergognosa. Si veda P. Damiani, Lettere, III, lettera 66, pp. 376-379.

12 G. Diacono, Istoria Veneticorum, pp. 198-99.

${ }_{13}$ MGH, DD, 5, Die Urkunden Heinrichs III, pp. 74-75.

${ }_{14}$ MGH, DD, 6/1, Die Urkunden Heinrichs IV, p. 600.

${ }^{15}$ F. Corner, Ecclesiae Venetae, XIII, p. 360. 
che lo inseriva nella complessa trama politica e diplomatica tessuta dai dogi. Nei diplomi imperiali veniva registrata la ragione delle visite a San Zaccaria, che era essenzialmente religiosa e simbolica: gli imperatori vi si recavano per pregare (ibi causa orationis presentes fuerunt). Dunque il monastero, forte del prestigio che derivava dal suo legame col doge, dalle sue ricchezze materiali e dal suo reclutamento aristocratico, rappresentava il luogo sacro in cui si dava corpo e forma simbolica alla potenza del doge e dell'intera città che il doge rappresentava. Fin dalla sua fondazione ebbe la funzione di preservare la gloria e la memoria del potere centrale, che era la potenza stessa di Venezia.

L'importanza di San Zaccaria nel processo politico di consolidamento dell'ufficio ducale e, di conseguenza, nell'intero panorama cittadino, era riflessa nel reclutamento delle sue monache e anzitutto nella scelta delle badesse: per quanto le fonti consentono di capire, grazie all'insolita ricchezza di indicazioni antroponomastiche che caratterizza la documentazione del nostro cenobio già a partire dall'XI secolo, la maggior parte delle badesse proveniva dall'aristocrazia veneziana e un certo numero di esse appartennero, fino oltre la metà del XII secolo, alle stesse famiglie dei dogi in carica; spesso erano loro figlie o sorelle. A titolo di esempio, Giovanna Parteciaco, attestata negli anni 878-880, era figlia del doge Orso I Parteciaco; Giovanna Candiano (963) era la moglie ripudiata del doge Pietro IV Candiano, mentre Maria Falier (1098) era anch'essa figlia del doge omonimo Vitale I Falier ${ }^{16}$. Casi di badesse imparentate coi dogi si registrarono anche nel XII secolo. Vita e Nella Michiel occuparono la carica abbaziale senza interruzione dal 1116 al 1141; il loro parente Domenico Michiel fu doge dal 1118 al 1130, e un altro Michiel, Vitale II, dal 1156 al 1172. Sulla base di queste scansioni cronologiche, che non possono essere casuali, si può persino ipotizzare che controllare l'abbaziato di San Zaccaria potesse essere uno dei passi che conducevano alla carica ducale, ma anche che la comunità femminile riuscisse, in certi momenti, a giocare un proprio ruolo attivo sullo scacchiere politico cittadino ai più alti livelli, e non fosse sempre e soltanto una pedina nelle mani delle famiglie. È comunque evidente che dietro alla scelta della badessa di San Zaccaria vi era una precisa strategia politica, attuata dalle famiglie ducali e da quelle che insieme a loro governavano la città, di occupazione sistematica del seggio abbaziale utilizzato come strumento di consolidamento e di appoggio dell'autorità centrale. San Zaccaria non era soltanto il monastero del doge, ma era il monastero di un intero gruppo dirigente, il quale per altro era impegnato nel faticoso tentativo di stabilizzare la propria distinta identità nel grande rimescolamento delle

16 I. Fees, Le monache di San Zaccaria, pp. 45-46; G. Rösch, Der Venezianische Adel, pp. 200-201. 
famiglie emergenti e di quelle in declino. Dal canto suo, la comunità, contando forse sulle doti politiche di qualche sua badessa particolarmente abile, dinamica e ben inserita nei networks cittadini ${ }^{17}$, poté consolidare ulteriormente il suo primato tra le fondazioni religiose veneziane prendendo decisioni autonome anche di natura economica e patrimoniale. Non si deve dimenticare che il prestigio di questo come degli altri cenobi femminili -ma analogo discorso si può fare ovviamente per quelli maschili- veniva misurato e pesato non soltanto sui simboli, ma anche sulla ricchezza patrimoniale. I cenobi veneziani si caratterizzavano nel loro complesso per il fatto di possedere la maggior parte dei propri beni al di fuori del ducato, sia in Terraferma sia nelle colonie della Romània ${ }^{18}$. I due femminili più antichi che qui si considerano disponevano però, oltre che di proprietà agricole nella Terraferma, di un numero notevolissimo di case e botteghe in città, tutte situate nelle parrocchie più vicine al cuore commerciale e politico veneziano, Rialto e San Marco ${ }^{19}$, che venivano concesse in affitto. Questi beni erano frutto certo di donazioni pie, ma soprattutto di una strategia patrimoniale di successo, attuata pazientemente dalle badesse nel corso del tempo, che avevano puntato a inserire il proprio ente nel mercato immobiliare cittadino, particolarmente vivace e redditizio. La disponibilità di così tanti edifici offriva inoltre una diversa, ma non meno importante occasione di stringere legami nuovi o di consolidare quelli più antichi con la multiforme società veneziana, con ricche famiglie aristocratiche e mercantili $\mathrm{o}$ anche con gruppi familiari di più modesta estrazione, ma economicamente molto attivi.

Nell'arco di tempo che qui si considera il mutamento istituzionale e sociale più importante fu la nascita del comune, il Commune Veneciarum, nel $1141^{20}$. Al di là delle diverse accentuazioni di questo o di quell'aspetto del processo che inserì Venezia nel solco delle altre città comunali dell'Italia centrosettentrionale, ciò che qui interessa sottolineare è il fatto che il sorgere dell'istituzione-comune determinò un sensibile aumento del numero di fami-

17 Sull'importanza per i cenobi, sia femminili che maschili, di una dirigenza monastica -a partire dall'abate, al cellerario, al priore ecc.- capace di attuare strategie di crescita per l'intera comunità si rimanda a A. Rapetti, Monachesimo medievale, pp. 71-119.

18 Sul tema si veda E. Orlando, "Ad profectum patriae". La proprietà ecclesiastica veneziana; specificamente sui beni di San Zaccaria, K. Modzelewski, Le vicende della "pars dominica".

19 F. Masé, Patrimoines immobiliers, p. 64.

20 G. Cracco, Società e stato nel medioevo veneziano, pp. 66-90; cfr. anche S. Gasparri, Dagli Orseolo al comune, pp. 818-820; A. Castagnetti, Il primo comune, pp. 81-130. A proposito dei mutamenti sociali di questi anni, G. Cracco, L'età del comune, p. 11, sottolinea che l'avvento del comune comportò "lo scatenarsi di aspirazioni, tensioni e conflitti (...). Ai vertici della società la concorrenza comportò il declino di vecchie stirpi ducali e l'ascesa di famiglie che non avevano affatto una tradizione ducale". 
glie che partecipavano al governo della città; si trattava di famiglie le quali, grazie alle nuove ricchezze provenienti dal commercio internazionale, erano ora in grado di competere con le stirpi più antiche, la cui base economica era invece la proprietà fondiaria. Questo fu infatti un periodo di grande e rapido incremento della ricchezza veneziana grazie all'estendersi delle reti del commercio internazionale e, nel giovane comune, chi si arricchiva col commercio poteva aspirare a entrare nel gruppo dirigente, accanto o in sostituzione di antiche famiglie ${ }^{21}$. La nuova dinamica competitiva fu ulteriormente amplificata dal rapido declino dell'autorità politica del doge, il cui predominio, che si fondava sulle famiglie originarie, fu messo in discussione dalle trasformazioni in corso. Come si vedrà, questo declino ebbe profonde ripercussioni sulla natura dei suoi rapporti con San Zaccaria.

La nascita del comune provocò una trasformazione profonda anche del ruolo politico delle istituzioni religiose del dogado. Il clero veneziano si trovò escluso dall'esercizio attivo del potere. Il legame tra i dogi, le famiglie di tradizione ducale e i vescovi cittadini, titolari della cattedra di Castello, che era stato particolarmente stretto fino all'avvento del comune, dopo di allora si sciolse; e infatti si trovano anche popolari tra i vescovi di Castello della seconda metà del XII secolo. Molti monasteri a loro volta si trovarono subordinati in misura crescente all'autorità degli ordinari diocesani, secondo una dinamica di disciplinamento delle comunità cenobitiche che caratterizza l'intero XII secolo anche al di fuori del dogado ${ }^{22}$. Ma nonostante questa novità essi continuarono evidentemente a offrire alle famiglie potenti di più antica origine un sostegno alla loro preminenza politica, e a quelle in ascesa nel nuovo clima istituzionale, determinato dal sorgere del comune, uno strumento ancora efficace, anche se non esclusivo, naturalmente, di consolidamento delle loro ambizioni.

In questo quadro così mutevole, nel quale tutti i soggetti in competizione cercavano strumenti politici e simbolici per legittimare prerogative e aspirazioni, e dove la composizione del gruppo dirigente aristocratico continuò a cambiare durante tutto il XII e il XIII secolo per l'ingresso di nuove famiglie e la scomparsa biologica di altre, i principali monasteri cittadini svolsero anche un'altra funzione decisiva. Essi furono un luogo nel quale la concorrenza e i conflitti tra le famiglie più antiche, che controllavano il governo della città sin dalla sua origine, e quelle di più recente fortuna economica $\mathrm{e}$ politica trovavano possibilità di accomodamento e occasioni di ricomposizione.

${ }^{21}$ G. Rösch, Il gran guadagno, pp. 233-36; G. Rösch, Der Venezianische Adel, p. 85-86.

22 In riferimento al clero e all'episcopato $c f r$. G. Rösch, Der Venezianische Adel, p. 83; pp. 192-193. Si vedano alcune considerazioni generali anche in G. Cracco, Società e stato, pp. 165-167. 
Nello stesso tempo, le famiglie aristocratiche che si legavano ai monasteri cittadini come patroni e benefattori se ne servirono come di un ambito politico -forse il solo disponibile a questo scopo-nel quale era possibile agire insieme alle altre, e non in concorrenza con esse. Soltanto nel sostegno ai monasteri più illustri l'aristocrazia parve muoversi come gruppo relativamente coeso, all'interno del quale la competizione per il potere poteva perdeva la sua carica eversiva e diventava quasi collaborativa nell'appoggio materiale e morale alle medesime comunità monastiche.

Come si concretizzava sul piano dei comportamenti questa tendenza collaborativa? In molti monasteri l'amministrazione dei beni e la difesa degli interessi sembra essere una attività condivisa contemporaneamente da diverse famiglie. È opportuno ricordare, per quanto possa sembrare ovvio, che anche nel ducato, come altrove, molti laici ebbero forti legami di patronato con chiese e monasteri. Tuttavia, l'evoluzione di molte fondazioni private fu radicalmente diversa da quanto si può osservare altrove. Nel volgere di poco tempo, come già si è in parte accennato, le cariche monastiche non furono più occupate dalle famiglie fondatrici ${ }^{23}$, e le comunità si conquistarono un ruolo sociale che consentì loro di presentarsi sulla scena politica cittadina come nuovi protagonisti. Il controllo patrimoniale di questi enti, che è notoriamente un elemento decisivo per comprendere la natura dei rapporti formalizzati tra monache e laici, non era appannaggio di un'unica famiglia, ma veniva condiviso tra famiglie diverse che, evidentemente, trovavano in quella funzione un'occasione di stringere nuove alleanze o di consolidare quelle già in atto. Verso la fine del Duecento, l'assetto politico di Venezia era in via di definizione. Al centro di tale struttura vi erano il Maggior Consiglio e la sua versione ridotta, il Minor Consiglio, ai quali erano riservate tutte le decisioni politicamente rilevanti per la vita del ducato. Oltre a questo, il processo di definizione dell'identità sociale del ceto dirigente giunse a conclusione, e gli scontri e i compromessi tra le due parti più influenti di quel ceto, i vecchi aristocratici e i mercanti, iniziarono a ridursi. Gli spazi per quelle pratiche politiche spregiudicate tipiche dell'epoca precedente, che avevano coinvolto profondamente anche gli enti ecclesiastici, erano ormai esauriti. Il risultato divenne chiaro nel 1297, quando fu approvata la legge costituzionale con la quale il gruppo delle famiglie che avevano o avevano avuto propri membri nel Consiglio arrogarono a se stesse il diritto esclusivo di governare Venezia. $\mathrm{Fu}$ grazie a questa legge, e a una serie di altri provvedimenti legislativi ema-

\footnotetext{
${ }^{23}$ Questa dinamica è evidente, oltre che per San Zaccaria, anche per San Lorenzo e per l'altro più antico monastero privato, quello maschile di Sant'Ilario (si veda sopra nota 3 e testo corrispondente). In ogni caso questi cenobi "non ebbero la forza di diventare elemento costituente di una signoria politica su base dinastica"; D. Rando, Una chiesa di frontiera, p. 60.
} 
nati nei trent'anni successivi, che il gruppo dirigente si trasformò in una vera e propria nobiltà definita su base ereditaria la quale si impose come organo di governo del ducato ${ }^{24}$.

A questo punto, l'utilità dei legami con i monasteri come forma di sostegno politico cominciò a diminuire, e contestualmente cominciò a venir meno la funzione politica dei monasteri.

Le famiglie nobili persero interesse verso l'amministrazione dei patrimoni monastici, perché non era più quello l'ambito in cui si poteva definire l'appartenenza o meno al gruppo dirigente. Ciò non vuol dire che non vi fosse più nessuna forma di controllo da parte dei laici sui cenobi, ma che tale controllo assunse caratteri diversi rispetto al passato, proprio in conseguenza delle mutate condizioni. Tra i protagonisti di questa nuova stagione di rapporti con i cenobi vi furono diversi ufficiali del governo, che comunque appartenevano alla nobiltà, visto che gli uffici pubblici erano riservati a quel ceto ${ }^{25}$. Ciò permise di inserire molte comunità in un sistema di aiuti pubblici di cui il veicolo principale furono le cosiddette grazie, provvedimenti liberali concessi dalle più importanti magistrature veneziane del tempo -ne avevano facoltà il doge e il Minor Consiglio, il Maggior Consiglio e la Quarantia- a favore di singoli individui o di persone giuridiche, con un campo di applicazione estremamente ampio. Le comunità entrarono attraverso queste grazie nel novero dei soggetti a favore dei quali lo stato elargiva somme di denaro destinate di solito a sovvenzionare lavori straordinari di restauro degli edifici monastici o di bonifica di terreni paludosi che si voleva rendere edificabili. Queste contribuzioni aumentarono rapidamente per numero e entità delle somme; a diverse comunità femminili furono concesse somme di denaro per esempio per ricostruire le mura degli edifici claustrali qui ceciderunt pro aqua magna (1300), o in subsidio reparacionis sui dormitorii (1303). Altre ottennero esenzioni dal pagamento dei dazi sul legname e sulle vettovaglie ${ }^{26}$.

${ }_{24}$ G. Cracco, Società e stato, pp. 290-351; G. Rösch, The Serrata of the Great Council, pp. 67-88. S. Chojnacki, La formazione della nobiltà, pp. 641-643.

${ }_{25}$ Tra gli altri si possono citare gli avvocati di San Zaccaria Pietro Michiel, che prestò la sua opera al monastero tra la fine del XII e l'inizio del XIII, ed è più volte attestato nello stesso periodo nelle liste dei giudici del comune, e Stefano Badoer, avvocato tra il 1227 e il 1235, forse advocator communis (G. Rösch, Der Venezianische Adel, pp. 93-96 e p. 101).

${ }^{26} \mathrm{Si}$ deve ricordare che, per la particolare conformazione idrogeologica della città e della laguna, gli interventi di bonifica degli spazi destinati alla costruzione e di restauro degli edifici danneggiati dalle acque erano continui ed estremamente dispendiosi, e tuttavia indispensabili per la sopravvivenza stessa della città. Anche i monasteri, molti dei quali dovettero addirittura abbandonare il luogo di insediamento originario e trasferirsi altrove per il crescente degrado dell' ambiente lagunare, erano impegnati in quest'opera, che può addirittura essere considerata il fondamento della coesione sociale del ducato, come sostiene E. Crouzet Pavan, Sopra le acque salse. Per i provvedimenti citati nel testo si veda E. Favaro (a cura di), Cassiere della bolla ducale. Grazie, n. 109, p. 27 (Santa Margherita di Torcello); n. 437, p. 101 (Santa Margherita 
Nel corso del XIII secolo cominciarono anche gli interventi delle autorità statali, che fino a quel momento non erano sembrate particolarmente attive nella vita dei cenobi del ducato, destinati a rafforzare il controllo pubblico sulle comunità e, per questa via, la coesione sociale. Furono coinvolti tanto quelli maschili quanto i femminili. Nel 1236, per esempio, l'abate di San Michele di Brondolo in diocesi di Chioggia -un grande monastero benedettino maschile fondato probabilmente nell'VIII secolo, che nel 1229 era stato riformato per ordine di Roma e incorporato all'ordine cistercense-fidelitatem sub juramento promisit communitati civitatis illius. La cosa aveva suscitato molte preoccupazioni al di fuori della laguna, tanto che il Capitolo generale dell'ordine era intervenuto per indagare sull'accaduto. È probabile che anche le badesse di San Matteo di Costanziaco e di Santa Margherita di Torcello, entrambe comunità cistercensi della laguna, assumessero lo stesso impegno ${ }^{27}$.

\section{LE POLITICHE DI RECLUTAMENTO DELLE MONACHE}

Come si è già detto, ed è del resto cosa nota, uno degli indicatori dei fenomeni relativi alle dinamiche sociali è la provenienza sociale delle monache professe nei principali monasteri. Se si fa di nuovo riferimento a San Zaccaria, si può osservare un cambiamento nella politica di reclutamento delle consorelle che si consolida gradualmente a partire dalla metà del XII secolo, cioè dopo l'avvento del comune. Anche gli altri antichi monasteri femminili, tra cui San Lorenzo, mostrano più o meno lo stesso mutamento, cioè l'apertura a monache provenienti dalle famiglie mercantili in ascesa. Fra i casi più noti c'è quello della monaca di San Zaccaria Ardefrosia, figlia di Romano Mairano, uno dei più ricchi mercanti attivi nella seconda metà del XII secolo, le cui fortune economiche subirono un grave colpo in seguito alla rappresaglia ordinata nel 1171 dall'imperatore Emanuele Comneno contro i veneziani residenti a Costantinopoli. Quanto rimase del suo patrimonio fu comunque sufficiente a garantire a sua figlia una dote adeguata, che le permise di essere accolta nel primo monastero cittadino, accanto alle figlie dell'aristocrazia, dove è attestata nel $1197^{28}$. Elemento significativo della valutazione è anche il numero di donne provenienti dallo stesso gruppo parentale che vivevano nelle stesse

di Torcello); per altri casi, sempre a favore di cenobi femminili, si vedano n. 224-225, p. 53-54 (San Giovanni di Torcello); n. 246, p. 58 (Sant'Antonio di Torcello).

27 S. Carraro, Tra sacro e quotidiano, p. 205; C. Moine, Chiostri tra le acque, pp. 27-29 e pp. 54-56.

${ }_{28}$ ASVE, Corporazioni religiose soppresse, San Zaccaria, b. 36 pergamene, 1197 luglio. Su Romano Mairano vedi G. Rösch, Der Venezianische Adel, p. 111. 
comunità monastiche. Nel nostro caso emerge che la maggior parte delle famiglie dell'aristocrazia maggiore sceglievano di monacare le proprie parenti in più di una comunità contemporaneamente. I Venier per esempio, una tra le famiglie più antiche della città, potevano vantare tra i loro parenti diverse monache e badesse in San Zaccaria, San Lorenzo, San Secondo, San Giovanni Evangelista e Sant'Antonio di Torcello, la cui presenza copre l'intero XIII secolo. Anche altre famiglie della stessa estrazione, che controllavano il potere fin dall'inizio, come i Contarini, i Michiel, i Morosini, i Dandolo, monacavano le proprie parenti in tre o quattro monasteri contemporaneamente. Ma ovviamente non esisteva una regola generale, perché le scelte dipendevano in larga parte dalle contingenze, dalle occasioni che si presentavano e, non ultimo, dalle necessità imposte dal gioco politico. Infine, le scelte delle famiglie dipendevano certamente anche da fattori del tutto esogeni ma non ininfluenti, quali la presenza di donne da monacare e la disponibilità di denaro per dotarle. Di conseguenza si trovano comportamenti anche molto differenti tra loro: per esempio non ci sono notizie di donne delle famiglie Tiepolo, Badoer e Viaro -famiglie la cui influenza politica si stava imponendo in quei decenni grazie al loro successo nel commercio ${ }^{29}$ - che scegliessero la vita consacrata a Venezia, ma in ogni caso non persero il collegamento con comunità più o meno importanti attraverso l'ufficio di procuratore monastico ${ }^{30}$.

Le differenze nelle scelte operate devono essere lette alla luce degli aspri conflitti che continuamente scuotevano il gruppo dirigente, come differenti strategie messe in atto dalle varie famiglie -coi mezzi di cui ciascuna disponeva- per raggiungere un nuovo equilibrio nella spartizione del potere. Anche da questo punto di vista i monasteri femminili parevano essere un ambiente favorevole allo scioglimento delle tensioni interne. Molte famiglie rafforzarono il controllo su di essi non con l'esclusione, ma con l'inclusione, favorendo a questo scopo un reclutamento molto largo. Viceversa, quando le strategie familiari si concentravano su un unico ente, le difficoltà facilmente si moltiplicavano e i conflitti potevano nuovamente scoppiare. I Loredan, per esempio, sembra preferissero concentrare le monacazioni delle loro parenti a Sant'Antonio di Torcello, ma come è facile comprendere, una scelta così concentrata, quale che ne fossero le ragioni, comportava anch'essa una percentuale di rischio. Molte monache Loredan erano entrate nella seconda metà

${ }^{29}$ G. Cracco, Società e stato, pp. 66-90; K. Takada, Aspetti della vita parentale, pp. 5-29.

${ }^{30}$ Limitandoci ancora una volta ai cenobi femminili: per esempio Marino Tiepolo, avvocato di San Lorenzo nel 1230 (ASVE, Corporazioni religiose soppresse, San Lorenzo, b. 22); Tommaso Viaro fu procuratore a San Servolo negli anni Sessanta del Duecento (ASVE, Corporazioni religiose soppresse, Santa Maria dell'Umiltà, b. 1 pergamene, San Servolo; G. Rösch, Der Venezianische Adel, pp. 219 e sgg.; p. 227); su Pietro e Stefano Badoer si vedano note 24 e 55. 
del Duecento a Sant'Antonio di Torcello; nel 1297 ve ne erano ben sei contemporaneamente, una delle quali era badessa ${ }^{31}$. Inoltre Giovanni Loredan, fratello della badessa Agnese e della monaca Marchesina, era il procuratore della comunità ${ }^{32}$. Nella stessa comunità vi erano anche diverse monache della famiglia Venier, della quale pure si è detto poco sopra, che era evidentemente poco disposta a concedere il completo controllo della comunità alle Loredan. Di lì a qualche anno scoppiò una violenta disputa a proposito dell'elezione della nuova badessa che vide schierarsi due fazioni contrapposte, con le Loredan da una parte e le Venier dall'altra, a sostegno ciascuna di una propria candidata; entrambe le fazioni, grazie anche alla chiamata in causa di vescovi e patriarchi, furono abbastanza forti da impedire di fatto l'elezione per un decennio, suscitando grande riprovazione fra le gerarchie ecclesiastiche veneziane. La soluzione fu trovata in un compromesso che permise l'elezione dapprima, nel 1320, di Beriola Venier la quale, una volta morta, fu sostituita da Fantina Loredan ${ }^{33}$.

Il caso di Giovanni Loredan consente di prendere in considerazione un'altra delle possibilità di cui disponevano i nobili laici, oltre alla monacazione di figlie e sorelle, di collegarsi a chiese e monasteri: occupare l'ufficio di avvocato e procuratore. Anche se questo ufficio non fu mai, a Venezia, appannaggio esclusivo delle famiglie dei fondatori, è però altrettanto chiaro che la scelta degli uomini che dovevano ricoprire una funzione delicata non veniva certo lasciata al caso: infatti, gli avvocati e i procuratori monastici provenivano sistematicamente da famiglie dell'aristocrazia cittadina, i casi di forestieri incaricati della rappresentanza di qualche importante cenobio veneziano furono sempre una rara eccezione ${ }^{34}$. La candidatura dei propri membri come avvocati o procuratori monastici veniva avanzata in tutte le occasioni che si presentavano, senza specifiche preferenze -almeno in apparenza- per un singolo cenobio, ma badando anzitutto al ruolo occupato dall'ente nel sistema politico e sociale cittadino. Per certi versi pare che le funzioni di avvocato e di procuratore monastico, che impegnavano per anni gli uomini più in vista della città a favore prima dell'uno, poi dell'altro cenobio, avessero assunto nel Duecento un carattere quasi professionale. Sommando le diverse funzioni, una famiglia, magari nel giro di pochi anni, poteva creare con un solo proprio esponente o con alcuni suoi membri legami di rilievo con più

\footnotetext{
${ }^{31}$ Le monache si chiamavano Agnese, Alice, Alvira, Antonia, Fontana, Marchesina: ASVE, Corporazioni religiose soppresse, Sant'Antonio di Torcello, b. 1 pergamene, 1297 maggio 14.

${ }^{32}$ ASVE, Corporazioni religiose soppresse, Sant'Antonio di Torcello, b. 1 pergamene, 1283 gennaio 3 .

${ }^{33}$ F. Corner, Ecclesiae Torcellanae, I, pp. 144-145; 160-164.

${ }^{34}$ G. Rösch, Der Venezianische Adel, p. 202.
} 
di un monastero. I Badoer, negli anni Venti e Trenta del Duecento, facevano donazioni a San Giovanni Evangelista tramite Giacomo ed erano presenti a San Zaccaria dove Stefano Badoer era avvocato ${ }^{35}$. Si trattava di un sistema di spartizione del potere che consentiva da un lato il controllo delle fondazioni monastiche, dall'altro garantiva un equilibrio abbastanza stabile nei rapporti di forza tra le varie famiglie interessate, e tra queste vi erano sempre le più importanti e prestigiose.

Le funzioni di questi procuratori erano molteplici: in generale dovevano curare gli affari della comunità e aiutarla in tutte le possibili necessità. Anzitutto dovevano rappresentarla in sede legale e giudiziaria, davanti alle autorità pubbliche, $\mathrm{e}$, in generale, dovevano vegliare sulla buona amministrazione dei beni, ma spesso intervenivano anche con sovvenzioni in denaro. Particolarmente generosa si dimostrò la famiglia Ziani, che diede due dogi a Venezia, Sebastiano (1172-1178) e Pietro (1205-1228). Le cronache del tempo elogiano la devozione di Pietro Ziani, che si dice si alzasse di notte per pregare, e ne ricordano le ricche donazioni a favore di enti maschili e femminili ${ }^{36}$. Pietro e suo figlio Marco furono per molti anni advocati del potente monastero maschile di San Giorgio Maggiore, e in più di un'occasione Pietro fu testimone in atti riguardanti San Zaccaria, al quale donò anche un terreno di pregio vicino a San Marco; a San Zaccaria sono inoltre attestate, tra la fine del XII e il XIII secolo, almeno tre monache Ziani ${ }^{37}$. La capacità di diversificare, per così dire, gli interessi famigliari nei riguardi degli enti ecclesiastici è ben evidente nel caso di Giovanni Barozzi, che dal 1210 al 1216 fu procuratore di San Zaccaria ${ }^{38}$, dove, tra il 1195 e il 1227 visse una monaca Agnese della stessa famiglia. Per meglio valutare il livello del suo impegno politico, basti infine ricordare che fu anche giudice nel 1208, sotto il governo di Pietro Ziani ${ }^{39}$.

Da queste brevissime biografie si può dedurre quanto importanti fossero le istituzioni cenobitiche non solo come terreno di composizione dei conflitti interni alle famiglie eminenti. Occupare le cariche amministrative di un prestigioso monastero, maschile o femminile, condividendo la responsabilità con altre stirpi potenti, significava per molte famiglie segnalare la propria appartenenza al gruppo dominante della città, per altre la volontà di raggiungere

${ }^{35}$ S. Giovanni Evangelista, (1225); ASVE, Corporazioni religiose soppresse, San Zaccaria, b. 8 pergamene, 1227-1232. Anche Stefano Badoer ebbe una lunga carriera politica, comprendente molteplici incarichi di podestà tra l'altro di Padova e di Ferrara (G. Cracco, Società e stato nel medioevo, p. 127; p. 156); L. Lanfranchi, San Giovanni Evangelista.

${ }^{36}$ I. Fees, Ricchezza e potenza, pp. 418-32.

37 Ibidem, pp. 299-304.

38 ASVE, Corporazioni religiose soppresse, San Zaccaria, b. 7 pergamene, 1210 aprile 30; b. 12 pergamene, 1216 aprile 30 .

39 G. Cracco, Società e stato, p. 72. 
quello stesso gruppo. Ma esse offrivano anche un'occasione di apprendere le pratiche politiche e di governo da applicare poi nell'ambito civile; una 'palestra' in cui gli uomini destinati alla carriera pubblica potevano esercitare e mettere alla prova la loro capacità di essere effettivamente ceto dirigente.

\section{LE NUOVE FONDAZIONI}

Verso la fine del XII secolo apparvero anche nel dogado le primi avvisaglie di quei vasti movimenti religiosi di ispirazione pauperistica e assistenziale che andavano largamente diffondendosi in Europa, sotto forma di fondazioni di piccole comunità femminili, che sorgevano non nel centro cittadino, ma in maggioranza nelle isole della laguna, soprattutto in quelle più settentrionali. Erano comunità che esprimevano le tendenze spirituali allora in voga, anzitutto l'aspirazione al desertum, particolarmente evidente nella scelta dell'insediamento su isole remote e poco popolate -ma non certo deserte, visto che vi sorgevano delle chiese-, che le correnti della laguna ora erodevano ora sommergevano. I più attivi sostenitori di queste iniziative femminili furono i vescovi di Torcello, che incoraggiarono e favorirono il consolidamento istituzionale delle esperienze religiose individuali attraverso una prassi più volte ripetuta: alle donne venivano concesse certe chiese, probabilmente in decadenza, appartenenti al vescovo, perché vi creassero una comunità monastica. Una peculiarità da sottolineare è il fatto che le nuove comunità, malgrado l'evidente sintonia con le correnti del movimento pauperistico, finirono per adottare tutte la regola benedettina, probabilmente per le pressioni dei vescovi di Torcello che, come si è detto, erano i loro primi benefattori. Si è ipotizzato che dietro alle cessioni di chiese abbandonate vi fosse il tentativo di invertire o almeno rallentare i fenomeni di spopolamento che iniziavano a interessare quella parte della laguna ${ }^{40}$, ma non si deve trascurare il fatto che la maggior parte di queste iniziative ebbe origine in una tensione religiosa e spirituale delle protagoniste, non certo in interessi di tipo economico o famigliare ${ }^{41}$. Non fu forse un caso che le famiglie veneziane, in un primo tempo, non si interessassero a questa novità, nonostante alcune delle comunità insulari fossero state fondate o beneficiate da donne dell'aristocrazia È il caso di Ginevra Gradenigo, appartenente a una delle famiglie più antiche e potenti di Venezia, che nel 1187 donò a Giacomina de Bonci il terreno sul quale sarebbe sorto il monaste-

40 L'interpretazione, molto nota, di cui si dice nel testo è sostenuta da E. Crouzet Pavan, Torcello, pp. 102-106.

${ }^{41}$ S. Carraro, Tra sacro e quotidiano, pp. 107-35; G. Spinelli, I monasteri benedettini, pp. 109-33. 
ro di Santa Maria degli Angelii ${ }^{42}$. Nel 1218, Maria da Canal e Maria Zancarolo, entrambe di famiglia aristocratica, furono le fondatrici del monastero di San Matteo nell'isola di Costanziaco, e qualche anno dopo Maria, detta Contessa, e Endrebona Dolfin promossero -non senza difficoltà- il passaggio di San Matteo all'ordine cistercense ${ }^{43}$. Le istanze religiose anche molto personali che stavano dietro a queste iniziative monastiche emergono con grande evidenza nella storia dell'appena citata Endrebona. Vedova di Pietro Michiel, che era stato avvocato di San Zaccaria nel $1185^{44}$, ciononostante Endrebona preferì diventare monaca in una remota isola della laguna anziché nel confortevole ed esclusivo cenobio dell'aristocrazia veneziana, cui apparteneva per nascita e per matrimonio. Un altro episodio: intorno al 1220 Agnese Longo, il cui marito era un importante mercante, partecipò alla costruzione degli edifici di Santa Margherita di Torcello ${ }^{45}$. Benché tutte queste fondazioni fossero dovute all'iniziativa di gruppi molto piccoli di mulieres religiosae, spesso non più di due o tre donne, esse cominciarono rapidamente a crescere e nel corso del Duecento raggiunsero dimensioni ragguardevoli; si trattò dunque di iniziative di successo in quanto a capacità di attrarre sia nuove vocazioni sia donazioni di terre ${ }^{46}$, in particolare quelle fondate da aristocratiche come la Gradenigo.

Abbastanza rapidamente si manifestò un certo interesse del patriziato verso questi lontani cenobi, del tutto analogo a quello che esso manifestava per le fondazioni più antiche e prestigiose. Dalla seconda metà del XIII secolo i monasteri della laguna iniziarono a reclutare un numero discreto di donne aristocratiche, anche se, naturalmente, in misura decisamente inferiore a quelle che entravano nelle comunità più antiche. Le monache, pur nell'isolamento fisico dell'isola, furono capaci di creare relazioni economiche ben al di fuori della laguna, riuscendo così a estendere il patrimonio delle comunità sulla terraferma. Oltre a questo, alcuni influenti personaggi pubblici si offrirono come procuratori e advocati di tutelare gli interessi legali ed economici delle monache. Gradualmente queste lontane comunità furono assimilate, nelle forme organizzative e nelle consuetudini di vita, a quelle cittadine, grazie agli interventi sia delle autorità ecclesiastiche che di quelle civili. Il reclutamento divenne sempre più aristocratico, nel senso che un numero crescente di famiglie

${ }^{42}$ F. Corner, Ecclesiae Torcellanae, I, p. 261.

${ }^{43}$ Ibidem, pp. 316-19; C. Moine, Chiostri tra le acque, pp. 27-29. Sulle famiglie delle fondatrici G. Rösch, Der Venezianische Adel, ad indicem.

${ }^{44}$ ASVE, Corporazioni religiose soppresse, San Zaccaria, b. 12 pergamene, 1203 giugno.

${ }^{45}$ S. Carraro, Tra sacro e quotidiano, p. 195; C. Moine, Chiostri tra le acque, pp. 54-56.

46 Per esempio, San Lorenzo di Ammiana venne fondata nel 1185 da due donne, Berta e Agnese. Nel 1252 le monache erano salite a diciassette: L. Lanfranchi (a cura di), San Lorenzo di Ammiana, pp. 81-83. ASVE, Corporazioni religiose soppresse, Santa Maria degli Angeli, b. $11 \mathrm{~b}, 1252$, luglio 29 . 
nobili inviò le proprie figlie in questi cenobi, che erano ormai altrettanto attraenti di quelli di Venezia. Da parte loro i vescovi di Torcello riuscirono ad adeguarne l'assetto istituzionale alle direttive generali provenienti da Roma, omologando lo stile di vita al monachesimo benedettino tradizionale e cancellando in questo modo anche il ricordo dell'originale ispirazione che li aveva fatti nascere.

Se si considerano i bacini geografici del reclutamento nei monasteri della laguna, si nota che la maggioranza delle monache professe erano veneziane; esaminando l'origine delle converse è invece facile osservare che la maggior parte di loro provenivano dalla terraferma, in particolar modo da quelle località in cui i monasteri avevano i loro patrimoni (quindi per esempio dalle zone di Padova e di Treviso), e dove a volte le converse risiedevano. I dati appena citati testimoniano di una notevole attenzione da parte delle famiglie veneziane nel controllo dei flussi di accesso alle comunità femminili della laguna, così come già avveniva per quelle di Venezia, che senza alcun dubbio era dettata dalla volontà di evitare qualsiasi potenziale interferenza nel rapporto con le monache da parte di forestieri. Un elenco di monache di San Zaccaria del 1195 è, sotto questo profilo, impressionante: delle oltre quaranta donne citate, la grande maggioranza apparteneva alla nobiltà e tutte le poche altre erano comunque originarie del ducato ${ }^{47}$. Questa cura e questa abilità nel regolare il reclutamento fanno ben capire quanto i monasteri femminili fossero, per l'aristocrazia, una parte costitutiva della sua coscienza di ceto. L'origine esclusivamente cittadina delle monache di monasteri, i quali tuttavia erano concretamente e massicciamente presenti, con i loro patrimoni fondiari, in aree esterne al ducato, può essere letto anche come segnale della capacità dei veneziani di estendere con molti mezzi la loro influenza al di fuori della città. Le monache veneziane (e i procuratori veneziani) diventavano una presenza fisica, nelle zone della terraferma in cui avevano i loro beni, che probabilmente veniva percepita come un'incarnazione del potere politico della città. Quindi, oltre alle funzioni sociali e politiche interne alla società cittadina, di cui si è lungamente detto, essi avevano una funzione fondamentale di rappresentazione della potenza dello stato al di fuori dei confini ducali; erano insomma una componente importante del ben conosciuto 'mito' di Venezia ${ }^{48}$.

Le fondazioni lagunari divennero nel Duecento oggetto delle cure amministrative dei nobili veneziani, come già lo erano quelle della città. Non è privo di significato che, in molti casi, furono gli uomini più attivi e influenti nella vita politica e nelle alte magistrature dello stato, spesso impegnati in

${ }^{47}$ G. Rösch, Der Venezianische Adel,p. 201; I. Fees, Le monache di San Zaccaria, pp. 35-43.

${ }^{48}$ Sul mito di Venezia si veda G. Fasoli, Nascita di un mito, pp. 445-472; E. Muir, Civic Ritual, pp. 17-75. 
carriere prestigiose, a svolgere le funzioni avvocatizie. Si può ricordare Andrea Zeno che, negli anni in cui era membro del Minor consiglio, agiva come procuratore di Sant'Antonio di Torcello $(1280)^{49}$, monastero nel quale, negli ultimi trent'anni del Duecento, vivevano almeno tre monache della famiglia. Un altro illustre Zeno, Ranieri, uomo politico dalla lunga e movimentata carriera, coronata dall'elezione a doge nel 1253, promosse la fondazione della comunità femminile cistercense di Santa Maria della Celestia (1237) mentre era podestà di Piacenza ${ }^{50}$; dieci anni dopo agiva come advocatus di San Lorenzo di Ammiana, istituito nel 1185 da due donne con il sostegno del vescovo di Torcello $^{51}$. Nel suo testamento del 1268, lo Zeno legò una somma consistente a un altro cenobio di recente fondazione, Santa Maria dei Crociferi, nato come ente assistenziale ${ }^{52}$. L'uomo era particolarmente influente nella politica veneziana: iniziò la carriera come podestà di Pola nel 1225, e negli anni successivi fino al 1240 ricoprì il ruolo di podestà in molti comuni italiani ${ }^{53}$. Nel 1245 rappresentò Venezia al concilio di Lione ${ }^{54}$ e infine divenne doge. Anche gli esponenti della famiglia Badoer si dimostrarono aperti alle novità religiose e istituzionali che apparivano sulla laguna. Se Stefano Badoer, tra 1'altro podestà di Padova nel 1231, e di Ferrara nel 1240, parve voler calcare le orme del suo antenato Pietro e, come quello, fu avvocato di San Zaccaria dal 1227 al $1232^{55}$, il suo parente Giovanni figlio di Giacomo si fece una fama come fondatore e benefattore di chiese e monasteri, mostrando una particolare preferenza per i nuovi ordini mendicanti e in particolare per i frati e le sorores Minori, che negli anni Trenta del Duecento si stavano affacciando in città. Nel 1234 Giovanni donò ai frati un terreno sul quale, di lì a poco, iniziò la

\footnotetext{
${ }^{49}$ ASVE, Corporazioni religiose soppresse, Sant'Antonio di Torcello, b. 1 pergamene, 1280 marzo 15

${ }^{50}$ S. Carraro, Tra sacro e quotidiano, pp. 143-147.

${ }^{51}$ ASVE, Corporazioni religiose soppresse, Miscellanea atti diplomatici e privati, b. 4, 1247 giugno 22 .

${ }_{52}$ Il testamento di Ranieri Zeno è pubblicato in J. Bernardi (a cura di), Antichi testamenti, pp. 14-18.

${ }^{53}$ Lo Zeno fu podestà a Chioggia (1228), Treviso (1233), Oderzo (1235), Piacenza (1237, Bologna (1239) e Fermo (1240). ASVE, Corporazioni religiose soppresse, San Matteo di Mazzorbo, b. 1 pergamene, 1228 giugno 8; San Michele in isola di Murano, b. 8, 1235 novembre 16; San Daniele, b. 2 pergamene, 1235 dicembre 12; G. Cracco, Società e stato, pp. 173-174; G. Rösch, Der Venezianische Adel, pp. 145-146. Sui podestà veneziani si veda anche J.-C. Maire Vigueur, Reclutamento e circolazione degli ufficiali.

${ }^{54}$ Durante il viaggio di ritorno fu catturato dall'imperatore Federico II, che lo costrinse ad assicurargli l'appoggio e l'aiuto del Commune Veneciarum contro i suoi avversari: P. Sambin, Problemi politici, pp.18-24.

${ }_{55}$ ASVE, Corporazioni religiose soppresse, San Zaccaria, b. 8 pergamene, 1227 novembre 30; 1232 aprile 30. Un altro Pietro Badoer era stato ugualmente avvocato di San Zaccaria durante il ventennio 1130-1150 (M. Pozza, I Badoer, p. 20).
} 
costruzione della chiesa conventuale dei Minori, Santa Maria dei Frari; nel 1236 avrebbe donato un altro appezzamento paludoso alle sorores minores insediate a Santa Chiara. Giovanni e i suoi discendenti rimasero da allora legati all'ordine francescano, e in particolare ai Frari, la cui costruzione terminò nel 1250 e dove volle essere sepolto ${ }^{56}$.

\section{CONCLUSIONI}

Si potrebbe fare altri esempi, a ulteriore conferma di quanto già è emerso dai casi appena ricordati, cioè come nel Duecento diverse famiglie si servissero delle relazioni con i monasteri per rafforzare la loro posizione politica; sul piano individuale poi, lo stretto legame con le comunità, che si esplicava nell'ufficio di advocatus o di procuratore, serviva anche per consolidare e dare smalto alle carriere politiche di questi brillanti 'grand commis' ante litteram, come nel caso di Ranieri Zeno. Non si spiegherebbe altrimenti perché uomini attivi nelle alte magistrature dello stato e spesso impegnati in carriere prestigiose continuassero anche nel Duecento, come in passato, ad occuparsi personalmente dell'amministrazione dei cenobi.

Se la novità politica e istituzionale, a metà del XII secolo, del commune Veneciarum e il graduale emergere, dall'antica élite cittadina legata al doge, di un ceto di governo in parte rinnovato nei suoi componenti e soprattutto dotato di nuovi strumenti per affermarsi, spinse le fondazioni monastiche, comprese quelle più potenti, al di fuori della lotta politica, questo non spezzò il loro antico legame con l'aristocrazia cittadina. Per tutto il lungo periodo compreso tra la nascita del comune e la 'serrata' del 1297 i rapporti con monasteri antichi e recenti continuarono ad essere uno strumento efficace, anche se non il solo, nel processo di costruzione dell'identità delle singole famiglie e di consolidamento della loro appartenenza al gruppo dominante non ancora definito giuridicamente. Quindi, nonostante l'utilità dei legami con i monasteri come forma di sostegno politico diminuisse gradualmente, le famiglie nobili non persero interesse verso l'amministrazione dei patrimoni monastici femminili, e continuarono a manifestarlo anche se in forme in parte diverse rispetto al passato, proprio in conseguenza delle mutate condizioni. Gli aristocratici coltivarono vecchie e nuove forme di collegamento. L'antica pratica di monacare le proprie parenti nei chiostri cittadini e della laguna si arricchì di una particolare attenzione ai bacini geografico e sociale di reclutamento

\footnotetext{
${ }^{56}$ S. Carraro, Tra sacro e quotidiano, pp. 148-151; M. Pozza, I Badoer, pp. 50-52.
} 
delle donne, i quali erano in prevalenza, tanto nel XII secolo quanto nel successivo, sia per i cenobi più antichi che per quelli più recenti, cittadino e 'magnatizio', se non esclusivamente aristocratico; questa leva di governo delle monache rimase saldamente nelle mani del gruppo dirigente veneziano, e non fu ovviamente per caso. Vi si aggiunse l'amministrazione dei patrimoni attraverso la carica di procuratori, praticata assiduamente soprattutto nel corso del Duecento da uomini che, nell'arco della loro vita, prestavano la propria opera in uno o più monasteri cittadini.

L'impegno profuso a favore delle comunità femminili anche in periodi di profondo mutamento politico e sociale fa ben capire come essi continuassero ad essere, per l'aristocrazia, una parte costitutiva della sua coscienza di ceto. I rapporti tra monache e laici sia nel XII che nel XII secolo si svilupparono, per la loro stessa importanza tanto per le prime quanto per i secondi, in forme sempre più chiare di collaborazione tra cenobi e famiglie, nell'ambito delle quali l'aristocrazia agiva come gruppo relativamente coeso, disposta a temperare la altrimenti consueta violenza della competizione per il potere e ad adottare un comportamento quasi cooperativo nell'appoggio materiale e morale alle medesime comunità monastiche. L'accordo probabilmente implicito tra famiglie normalmente concorrenti consentiva da un lato il controllo delle fondazioni monastiche, dall'altro metteva queste ultime al sicuro dai possibili danni derivanti dalla rivalità fra i loro protettori. Come già in passato, i cenobi femminili ricevevano a loro volta sostegno materiale e prestigio sociale dalla partecipazione delle famiglie eminenti alla vita delle monache, in un rapporto simbiotico e reciprocamente vantaggioso che fu la ragione della sua lunga durata pur in un'epoca di profonde trasformazioni. Il patronato di queste famiglie assicurava ai monasteri prestigio, ricchezza economica e rilevanza sociale e consentiva loro di rivendicare privilegi e tradizioni particolari che ne caratterizzavano il ruolo sociale all'interno di Venezia.

\section{BIBLIOGRAFIA CITATA}

Agazzi, Michela, Platea Sancti Marci. I luoghi marciani dall'XI al XIII secolo e la formazione della piazza, Venezia, Comune di Venezia, 1991.

Bernardi, Jacopo (a cura di), Antichi testamenti tratti dagli archivi della congregazione della carità di Venezia per la dispensa delle visite, Venezia, Tipografia di M.S. fra compositori e impressori, 1884, pp. 3-18.

Carraro, Silvia, Tra sacro e quotidiano. Il monachesimo femminile nella laguna di Venezia in epoca medievale (secoli IX-XIV), Milano, Università degli Studi, a.a. 2011-2012 (tesi di dottorato). 
Castagnetti, Andrea, Il primo comune, in Cracco, Giorgio; Ortalli, Gherardo (a cura di), Storia di Venezia dalle origini alla caduta della Serenissima, 2. L'età del comune, Roma, Istituto della Enciclopedia Italiana, 1995, pp. 81-130.

Chojnacki, Stanley, La formazione della nobiltà dopo la Serrata, in Arnaldi, Girolamo; Cracco, Giorgio; Tenenti, Alberto (a cura di), Storia di Venezia dalle origini alla caduta della Serenissima, 3. La formazione dello stato patrizio, Roma, Istituto della Enciclopedia Italiana, 1997, pp. 641-725.

Corner, Flaminio, Ecclesiae Torcellanae antiquis monumentis nunc etiam primum editis illustratae, III vols., Venezia, Typis Johannis Baptistae Pasquali, 1749.

Corner, Flaminio, Ecclesiae Venetae antiquis monumentis nunc etiam primum editis illustratae ac in decades distribuitae, 14 vols., Venezia, Typis Johannis Baptistae Pasquali, 1749.

Cracco, Giorgio, Società e stato nel medioevo veneziano (secoli XII-XIV), Firenze, Olschki, 1967

Cracco, Giorgio, L'età del comune, in Cracco, Giorgio; Ortalli, Gherardo (a cura di), Storia di Venezia dalle origini alla caduta della Serenissima, 2. L'età del comune, Roma, Istituto della Enciclopedia Italiana, 1995, pp. 1-30.

Crouzet Pavan, Elisabeth, Sopra le acque salse: espaces, pouvoir et société à Venise à la fin du Moyen Age, II vols., Roma, École française de Rome, 1982.

Crouzet Pavan, Elisabeth, Torcello. Storia di una città scomparsa, Roma, Jouvence, 2001.

Damiani, Pier, Lettere, a cura di Innocenzo Gargano, Nicolangelo D'Acunto, III, Roma, Città Nuova Editrice, 2002.

Da Mosto, Andrea, L'Archivio di Stato di Venezia. Indice generale, storico, descrittivo ed analitico, II, Roma, Biblioteca d'arte, 1940.

Diacono, Giovanni, Istoria Veneticorum, a cura di Luigi Andrea Berto, Bologna, Zanichelli editore, 1999.

Fasoli, Gina, Nascita di un mito, in Bocchi, Francesca; Carile, Antonio; Pini, Antonio Ivan (a cura di), Scritti di storia medievale, Bologna, La fotocromo emiliana, 1974, pp. 445-72.

Favaro, Elena (a cura di), Cassiere della bolla ducale. Grazie-Novus liber (1299-1305), Fonti per la storia di Venezia: Archivi pubblici, 1, Venezia, Il comitato editore, 1962.

Fees, Irmgard, Le monache di San Zaccaria a Venezia nei secoli XII e XIII, Venezia, Centro tedesco di studi veneziani, 1998.

Fees, Irmgard, Ricchezza e potenza nella Venezia medievale: la famiglia Ziani, Roma, Il Veltro 2006. 
Gasparri, Stefano, Dagli Orseolo al comune, in Benzoni, Gino; Menniti, Ippolito Antonio; Ruggini Cracco, Lellia (a cura di), Storia di Venezia dalle origini alla caduta della Serenissima, 1. Origini-età ducale, Roma, Istituto della Enciclopedia Italiana, 1992, pp. 781-830.

Lanfranchi, Luigi (a cura di), San Lorenzo di Ammiana, Fonti per la storia di Venezia: Archivi ecclesiastici, diocesi Torcellana, 2, Venezia, Alfieri, 1969.

Lanfranchi, Luigi (a cura di), San Giovanni Evangelista di Torcello, Fonti per la storia di Venezia: Archivi ecclesiastici, diocesi Torcellana, 2, Venezia, Alfieri, 1969.

Lowe, Kate, Power and institutional identity in Renaissance Venice: the female convents of S. Maria delle Vergini and S. Zaccaria, in Collegium. Studies across disciplines in the humanities and social sciences, 2. The trouble with ribs: women, men and gender in Early Modern Europe, Helsinki, Helsinki Collegium for Advanced Studies, 2007, pp. 128-152.

Maire Vigueur, Jean-Claude (a cura di), I podestà dell'Italia comunale, I, Reclutamento e circolazione degli ufficiali forestieri (fine XII sec.-metà $X I V$ sec.), II vols., Roma, Istituto storico italiano per il medioevo, 2000.

Masé, Federica, Patrimoines immobiliers ecclésiastiques dans la Venise médiéval (XIe-XVe siècle). Une lecture de la ville, Roma, École française de Rome, 2006.

Modzelewski, Karol, Le vicende della "pars dominica" nei beni fondiari del monastero di San Zaccaria di Venezia (secoli X-XIV), "Bollettino dell'istituto di storia della società e dello stato veneziano" 4 (1962), pp. 42-79; 5 (1963), pp. 15-63.

Moine, Cecilia, Chiostri tra le acque. I monasteri femminili della laguna nord di Venezia nel basso medioevo, Borgo San Lorenzo (FI), All'Insegna del Giglio, 2013.

Muir, Edward, Civic Ritual in Renaissance Venice, Princeton (NJ), Princeton University Press, 1981.

Orlando, Ermanno, "Ad profectum patriae". La proprietà ecclesiastica veneziana in Romània dopo la IV crociata, Roma, Istituto storico italiano per il medioevo, 2005.

Pozza, Marco, I Badoer. Una famiglia veneziana dal X al XIII secolo, Abano Terme, Francisci, 1982.

Pozza, Marco, Per una storia dei monasteri veneziani nei secoli VIII-XII, in Trolese, Francesco (a cura di), Il monachesimo nel Veneto medioevale. Atti del convegno di studi in occasione del millenario di fondazione dell'abbazia di S. Maria di Mogliano Veneto (Treviso), Cesena, Badia di Santa Maria del Monte, 1998, pp. 17-38. 
Rando, Daniela, Una chiesa di frontiera. Le istituzioni ecclesiastiche veneziane nei secoli VI-XII, Bologna, Il Mulino, 1994.

Rapetti, Anna, Monachesimo medievale. Uomini, donne, istituzioni, Venezia, Marsilio, 2005.

Rösch, Gerhard, Der Venezianische Adel, bis zur Schließung des Großen Rats. Zur Genese einer Führungsschicht, Sigmaringen, Thorbecke, 1989.

Rösch, Gerhard, Il gran guadagno, in Cracco, Giorgio; Ortalli, Gherardo (a cura di), Storia di Venezia dalle origini alla caduta della Serenissima, 2. L'età del comune, Roma, Istituto della Enciclopedia Italiana, 1995, pp. 233-59.

Rösch, Gerhard, The Serrata of the Great Council and Venetian society, 12861323, in Martin, John; Romano, Dennis (eds.), Venice Reconsidered: the history and civilization of an Italian City-State, 1297-1797, Baltimore (MD), Johns Hopkins University Press, 2000, pp. 67-88.

Sambin, Paolo, Problemi politici attraverso lettere inedite di Innocenzo IV, "Istituto Veneto di scienze lettere e arti" 31 (1955), pp. 18-34.

Spinelli, Giovanni, I monasteri benedettini fra il 1000 e il 1300, in Tonon, Franco (a cura di), La chiesa di Venezia nei secoli XI-XIII, Venezia, Studium cattolico veneziano, 1988.

Takada, Keiro, Aspetti della vita parentale della nobiltà veneziana nel Duecento. L'esempio della famiglia Viaro del ramo di San Maurizio, "Archivio Veneto" 180 (1995), pp. 5-29.

Fecha de recepción del artículo: noviembre 2013

Fecha de aceptación y versión final: marzo 2014 